\title{
Liouville type theorem for higher-order elliptic system with Navier boundary condition
}

Weiwei Zhao, Jinge Yang and Sining Zheng

\begin{abstract}
This paper deals with the Liouville theorem for a higher-order elliptic system in the half-space subject to the Navier boundary value conditions. We obtain this via establishing the Liouville type theorem for the equivalent integral system by the moving plane method.
\end{abstract}

Mathematics Subject Classification. Primary 35J58; Secondary $35 \mathrm{~J} 61$.

Keywords. Higher-order elliptic system, Liouville type theorem, Navier boundary value, Moving planes method in integral form, Rotational symmetry.

\section{Introduction}

In this paper, we establish a Liouville type theorem for the $2 m$-order elliptic equations coupled via the Navier boundary conditions in the half-space $\mathbb{R}_{+}^{n}=$ $\left\{x \in \mathbb{R}^{n}: x_{n}>0\right\}$ :

$$
\begin{cases}(-\Delta)^{m} u(x)=v^{\beta} & \text { in } \mathbb{R}_{+}^{n}, \\ (-\Delta)^{m} v(x)=u^{\alpha} & \text { in } \mathbb{R}_{+}^{n}, \\ u=\Delta u=\cdots=\Delta^{m-1} u=0 & \text { on } \partial \mathbb{R}_{+}^{n}, \\ v=\Delta v=\cdots=\Delta^{m-1} v=0 & \text { on } \partial \mathbb{R}_{+}^{n},\end{cases}
$$

where $m$ is a positive integer satisfying $0<2 m<n, \frac{n}{n-2 m}<\alpha, \beta \leq \frac{n+2 m}{n-2 m}$.

Various higher-order elliptic equations can be used to model complex spatio-temporal pattern formations $[26,28,33]$. They also appear in studying the so-called Paneitz-Branson operator and its generalizations, with important applications in mathematical physics $[4,12,14]$. There are many other applications of them for such as the hinged plate problems [25,34], the switched

This work is supported by the NNSF of China (11171048) and the STRP of Jiangxi Province (GJJ14759, 20142BAB211007). 
diffusion processes (in probability theory) [10], and the switching costs problems (in stochastic control) [18].

It is well known that in order to establish a priori estimates for nonlinear elliptic equations and systems of equations without variational structure, one usually uses the blow-up argument [16]. The argument, in turn, relies on nonexistence theorems for positive solutions of the limiting problems, i.e., Liouville type theorems. Thus, Liouville type theorems play a very important role in the study of nonlinear elliptic equations without variational structure.

It is known from $[11,24,27,31]$ that the whole space problem

$$
(-\Delta)^{m} u(x)=v^{\beta}, \quad(-\Delta)^{m} v(x)=u^{\alpha} \text { in } \mathbb{R}^{n}
$$

with $m=1$ does not admit positive classical solutions in the following cases:

(a) $\frac{1}{\beta+1}+\frac{1}{\alpha+1}>\frac{n-2}{n}$ and either $u, v$ are radial or $n=3$;

(b) $\max \left(\frac{2(\beta+1)}{\alpha \beta-1}, \frac{2(\alpha+1)}{\alpha \beta-1}\right) \geq n-2$;

(c) $\alpha, \beta \leq \frac{n+2}{n-2}$ with $\frac{\alpha+\beta}{2}<\frac{n+2}{n-2}$.

This Liouville type result with $m=1$ is still valid for the half space problem (1.1) under the same parameter regions (a)-(c) [3], and also has been extended to the biharmonic case with $m=2$ [13].

For the polyharmonic system $(1.2)(m \neq 1)$ in whole space, Guo, Liu, and Zhang [20] proved the following Liouville type results that the problem has no positive classical solutions in the following cases:

(a) $\alpha, \beta \geq 1$ with $\frac{\alpha+\beta}{2}>1, \frac{1}{\beta+1}+\frac{1}{\alpha+1}>\frac{n-2}{n}$, and $u, v$ are radial;

(b) $\alpha, \beta \geq 1$ with $\frac{\alpha+\beta}{2}>1,(n-2 m) \alpha<\frac{n}{\beta}+2 m$ or $(n-2 m) \beta<\frac{n}{\alpha}+2 m$;

(c) $1 \leq \alpha, \beta \leq \frac{n+2 m}{n-2 m}$ with $1<\frac{\alpha+\beta}{2}<\frac{n+2 m}{n-2 m}$.

In addition, Zhang [35] proved that if $n>2 m, \alpha, \beta \geq 1$ with $\frac{\alpha+\beta}{2}>1$, and $\frac{2 m(\beta+1)}{\alpha \beta-1}, \frac{2 m(\alpha+1)}{\alpha \beta-1} \in\left[\frac{n-2}{2}, n-2 m\right)$, then (1.2) has no positive classical solutions.

The half space problems have been thoroughly studied as well. The Liouville theorem for the Dirichlet type boundary conditions was shown in $[15,29,30]$ that if $u$ is a classical solution of

$$
\begin{cases}(-\Delta)^{m} u(x)=u^{p} & \text { in } \mathbb{R}_{+}^{n}, \\ u \geq 0 & \text { in } \mathbb{R}_{+}^{n}, \\ u=\frac{\partial u}{\partial x_{n}}=\cdots=\frac{\partial^{m-1} u}{\partial x_{n}^{m-1}}=0 & \text { on } \partial \mathbb{R}_{+}^{n},\end{cases}
$$

with $1<p<\frac{n+2 m}{n-2 m}$, then $u \equiv 0$. The Liouville theorem with the Navier boundary problem of higher order equation in half space was studied in [32]. Please see $[5,9,21]$ for the current and more general results on the Liouville theorem with the Navier boundary condition. Also mention the early important works $[1,2]$ of Berestycki, Capuzzo Dolcetta and Nirenberg on second order equations. 
In the present paper, instead of (1.1), we will at first establish a Liouville type theorem for the integral system

$$
\left\{\begin{array}{l}
u(x)=C_{n} \int_{\mathbb{R}_{+}^{n}}\left(\frac{1}{|x-y|^{n-2 m}}-\frac{1}{|\bar{x}-y|^{n-2 m}}\right) v^{\beta}(y) d y \\
v(x)=C_{n} \int_{\mathbb{R}_{+}^{n}}\left(\frac{1}{|x-y|^{n-2 m}}-\frac{1}{|\bar{x}-y|^{n-2 m}}\right) u^{\alpha}(y) d y
\end{array}\right.
$$

where $C_{n}>0, \bar{x}=\left(x_{1}, \ldots, x_{n-1},-x_{n}\right)$ is the reflection of the point $x$ about the $\partial \mathbb{R}_{+}^{n}$, and then prove that the two systems are equivalent each other under certain conditions. That is the following two theorems:

Theorem 1.1. Let $(u, v) \in L_{\mathrm{loc}}^{p}\left(\mathbb{R}_{+}^{n}\right) \times L_{\mathrm{loc}}^{q}\left(\mathbb{R}_{+}^{n}\right)$ be a positive solution of the system (1.3) with $p=\frac{\alpha-1}{\frac{n-2 m}{n} \alpha-1}$ and $q=\frac{\beta-1}{\frac{n-2 m}{n} \beta-1}$. If $\frac{n}{n-2 m}<\alpha, \beta \leq \frac{n+2 m}{n-2 m}$, then $(u, v) \equiv(0,0)$.

Theorem 1.2. Let $(u, v)$ be a classical positive solution of system (1.1) with $\alpha, \beta \geq 1$, then the differential system (1.1) is equivalent to the integral system (1.3).

Theorems 1.1 and 1.2 yield the main result of the paper:

Theorem 1.3. Under the conditions of Theorems 1.1 and 1.2, the classical positive solutions of system (1.1) must be trivial.

To prove Theorem 1.1, we will explore the moving plane method in integral forms by Chen-Li-Ou [8]. Corresponding to the half space problem (1.3), the Liouville type theorem to the whole space problem was established by Ma and Chen [22]. Refer to [7,19,23] and the references therein. Theorem 1.2 can be simply treated by using a technique introduced by Chen and Fang [9] for the scalar case of higher-order equations.

via the equivalent system coupled by $2 m$ elliptic equations of 2 -order and the related system of $2 m$ integral equations, .

This paper is arrange as follows. We will give some preliminaries in Sect. 2 , and then prove the main results of the paper in Sect. 3.

\section{Preliminaries}

We introduce a series lemmas for the integral system (1.3) as preliminaries, and let $C_{n}=1$ there for simplicity in the sequence.

Denote

$$
G(x, y)=\frac{1}{|x-y|^{n-2 m}}-\frac{1}{|\bar{x}-y|^{n-2 m}}, \quad x, y \in \mathbb{R}_{+}^{n},
$$

with $\bar{x}$ reflecting $x$ about the $\partial \mathbb{R}_{+}^{n}$. Let $x^{\lambda, 1}=\left(2 \lambda-x_{1}, x_{2}, \ldots, x_{n}\right)$ be the reflection of the point $x$ about the plane $T_{\lambda, 1}=\left\{x \in \mathbb{R}_{+}^{n} \mid x_{1}=\lambda\right\}$, and denote $u_{\lambda, 1}(x)=u\left(x^{\lambda, 1}\right), v_{\lambda, 1}(x)=v\left(x^{\lambda, 1}\right)$. Define $H_{\lambda, 1}=\left\{x \in \mathbb{R}_{+}^{n} \mid x_{1}<\lambda\right\}, H_{\lambda, 1}^{c}=$ $\mathbb{R}_{+}^{n} \backslash H_{\lambda, 1}$. Generally, some global integrability of solutions is required for the 
moving-plane method in integral form. In the present paper, the solutions are just assumed locally integrable. We employ the Kelvin transformation

$\bar{u}(x)=\frac{1}{\left|x-z^{0}\right|^{n-2 m}} u\left(\frac{x-z^{0}}{\left|x-z^{0}\right|^{2}}+z^{0}\right), \bar{v}(x)=\frac{1}{\left|x-z^{0}\right|^{n-2 m}} v\left(\frac{x-z^{0}}{\left|x-z^{0}\right|^{2}}+z^{0}\right)(2$

with $z_{0} \in \partial \mathbb{R}_{+}^{n}$. We have an equivalent integral system for (2.2):

Lemma 2.1. Let $(u, v)$ be a positive solution of system (1.3). Then $(\bar{u}, \bar{v})$ solves

$$
\left\{\begin{array}{l}
u(x)=\int_{\mathbb{R}_{+}^{n}} G(x, y) \frac{1}{\left|y-z^{0}\right|^{(n+2 m)-\beta(n-2 m)}} v^{\beta}(y) d y, \\
v(x)=\int_{\mathbb{R}_{+}^{n}} G(x, y) \frac{1}{\left|y-z^{0}\right|^{(n+2 m)-\alpha(n-2 m)}} u^{\alpha}(y) d y .
\end{array}\right.
$$

Proof. Notice

$$
\begin{aligned}
& \left|\left(\frac{x-z^{0}}{\left|x-z^{0}\right|}\right)\right| t-z^{0}\left|-\left(\frac{t-z^{0}}{\left|t-z^{0}\right|}\right)\right| x-z^{0}||^{2}=\left|\left(x-z^{0}\right)-\left(t-z^{0}\right)\right|^{2}=|x-t|^{2}, \\
& \left|\left(\frac{\bar{x}-z^{0}}{\left|\bar{x}-z^{0}\right|}\right)\right| t-z^{0}\left|-\left(\frac{t-z^{0}}{\left|t-z^{0}\right|}\right)\right| \bar{x}-z^{0}||^{2}=\left|\left(\bar{x}-z^{0}\right)-\left(t-z^{0}\right)\right|^{2}=|\bar{x}-t|^{2},
\end{aligned}
$$

and $\left|x-z^{0}\right|=\left|\bar{x}-z^{0}\right|$ for $z^{0} \in \partial \mathbb{R}_{+}^{n}$. Let $y=\frac{t-z_{0}}{\left|t-z^{0}\right|^{2}}+z^{0}$. We have for the solution $(u, v)$ of $(2.3)$ by a simple computation that

$$
\begin{aligned}
\bar{u}(x) & =\frac{1}{\left|x-z^{0}\right|^{n-2 m}} \int_{\mathbb{R}_{+}^{n}}\left(\frac{1}{\left|\frac{x-z^{0}}{\left|x-z^{0}\right|^{2}}+z^{0}-y\right|^{n-2 m}}-\frac{1}{\left|\frac{\bar{x}-z^{0}}{\left|\bar{x}-z^{0}\right|^{2}}+z^{0}-y\right|^{n-2 m}}\right) v^{\beta}(y) d y \\
& =\int_{\mathbb{R}_{+}^{n}}\left(\frac{1}{|x-t|^{n-2 m}}-\frac{1}{|\bar{x}-t|^{n-2 m}}\right) \frac{\bar{v}^{\beta}(t)}{\left|t-z^{0}\right|^{(n+2 m)-\beta(n-2 m)}} d t,
\end{aligned}
$$

and a similar result for $\bar{v}$.

Remark 1. Due to Lemma 2.1, it suffices to prove the Liouville type conclusion for the system (2.3).

Lemma 2.2. Let $(u, v)$ be a positive solution of (2.3). Then for any $x \in H_{\lambda, 1}$, we have

$$
\begin{aligned}
u(x)-u_{\lambda, 1}(x)= & \int_{H_{\lambda, 1}}\left[G\left(x^{\lambda, 1}, y^{\lambda, 1}\right)-G\left(x, y^{\lambda, 1}\right)\right] \\
& \times\left[\frac{v^{\beta}(y)}{\left|y-z^{0}\right|^{(n+2 m)-\beta(n-2 m)}}-\frac{v_{\lambda, 1}^{\beta}(y)}{\left|y^{\lambda, 1}-z^{0}\right|^{(n+2 m)-\beta(n-2 m)}}\right] d y, \\
v(x)-v_{\lambda, 1}(x)= & \int_{H_{\lambda, 1}}\left[G\left(x^{\lambda, 1}, y^{\lambda, 1}\right)-G\left(x, y^{\lambda, 1}\right)\right] \\
& \times\left[\frac{u^{\alpha}(y)}{\left|y-z^{0}\right|^{(n+2 m)-\alpha(n-2 m)}}-\frac{u_{\lambda, 1}^{\alpha}(y)}{\left|y^{\lambda, 1}-z^{0}\right|^{(n+2 m)-\alpha(n-2 m)}}\right] d y .
\end{aligned}
$$


Proof. By the first equation of (2.3),

$$
\begin{aligned}
u(x)= & \int_{H_{\lambda, 1}} G(x, y) \frac{v^{\beta}(y)}{\left|y-z^{0}\right|^{(n+2 m)-\beta(n-2 m)}} d y \\
& +\int_{H_{\lambda, 1}} G\left(x, y^{\lambda, 1}\right) \frac{v_{\lambda, 1}^{\beta}(y)}{\left|y^{\lambda, 1}-z^{0}\right|^{(n+2 m)-\beta(n-2 m)}} d y, \\
u_{\lambda, 1}(x)= & \int_{H_{\lambda, 1}} G\left(x^{\lambda, 1}, y\right) \frac{v^{\beta}(y)}{\left|y-z^{0}\right|^{(n+2 m)-\beta(n-2 m)}} d y \\
& +\int_{H_{\lambda, 1}} G\left(x^{\lambda, 1}, y^{\lambda, 1}\right) \frac{v_{\lambda, 1}^{\beta}(y)}{\left|y^{\lambda, 1}-z^{0}\right|^{(n+2 m)-\beta(n-2 m)}} d y .
\end{aligned}
$$

Since

$$
G(x, y)=G\left(x^{\lambda, 1}, y^{\lambda, 1}\right), G\left(x^{\lambda, 1}, y\right)=G\left(x, y^{\lambda, 1}\right), x, y \in H_{\lambda, 1}, x \neq y,
$$

we get (2.4) directly. The same is true for (2.5).

Denote $\Sigma_{\mu, n}=\left\{x \in \mathbb{R}_{+}^{n} \mid 0<x_{n}<\mu\right\}, T_{\mu, n}=\left\{x \in \mathbb{R}_{+}^{n} \mid x_{n}=\mu\right\}$, $\tilde{\Sigma}_{\mu, n}=\left\{x^{\mu, n} \mid x \in \Sigma_{\mu, n}\right\}, \Sigma_{\mu, n}^{c}=\mathbb{R}_{+}^{n} \backslash \Sigma_{\mu, n}$. The following lemma on the Green function $G(x, y)$ in $\Sigma_{\mu, n}$ was known.

Lemma 2.3. (Lemma 2.1 in [6])

(i) For any $x, y \in \Sigma_{\mu, n}, x \neq y$, we have

$$
\begin{aligned}
& G\left(x^{\mu, n}, y^{\mu, n}\right)>\max G\left(x^{\mu, n}, y\right), G\left(x, y^{\mu, n}\right), \\
& G\left(x^{\mu, n}, y^{\mu, n}\right)-G(x, y)>\left|G\left(x^{\mu, n}, y\right)-G\left(x, y^{\mu, n}\right)\right| .
\end{aligned}
$$

(ii) For any $x \in \Sigma_{\mu, n}, y \in \Sigma_{\mu, n}^{c}$, it holds that

$$
G\left(x^{\mu, n}, y\right)>G(x, y) \text {. }
$$

Lemma 2.4. Let $(u, v)$ be a positive solution of (1.3). For any $x \in \Sigma_{\mu, n}$, we have

$$
\begin{aligned}
& u(x)-u_{\mu, n}(x) \leq \int_{\Sigma_{\mu, n}} G\left(x^{\mu, n}, y^{\mu, n}\right)\left(v^{\beta}-v_{\mu, n}^{\beta}\right)(y) d y \\
& v(x)-v_{\mu, n}(x) \leq \int_{\Sigma_{\mu, n}} G\left(x^{\mu, n}, y^{\mu, n}\right)\left(u^{\alpha}-u_{\mu, n}^{\alpha}\right)(y) d y .
\end{aligned}
$$

Proof. We only deal with the first inequality. Since

$$
\begin{aligned}
u(x)= & \int_{\Sigma_{\mu, n}} G(x, y) v^{\beta}(y) d y+\int_{\Sigma_{\mu, n}} G\left(x, y^{\mu, n}\right) v_{\mu, n}^{\beta}(y) d y \\
& +\int_{\Sigma_{\mu, n}^{c} \backslash \tilde{\Sigma}_{\mu, n}} G(x, y) v^{\beta}(y) d y, \\
u_{\mu, n}(x)= & \int_{\Sigma_{\mu, n}} G\left(x^{\mu, n}, y\right) v^{\beta}(y) d y+\int_{\Sigma_{\mu, n}} G\left(x^{\mu, n}, y^{\mu, n}\right) v_{\mu, n}^{\beta}(y) d y \\
& +\int_{\Sigma_{\mu, n}^{c} \backslash \tilde{\Sigma}_{\mu, n}} G\left(x^{\mu, n}, y\right) v^{\beta}(y) d y,
\end{aligned}
$$


we have by Lemma 2.3 that

$$
\begin{aligned}
u(x)-u_{\mu, n}(x) & \leq \int_{\Sigma_{\mu, n}}\left[G\left(x^{\mu, n}, y^{\mu, n}\right)-G\left(x, y^{\mu, n}\right)\right] v^{\beta}(y) d y \\
& -\int_{\Sigma_{\mu, n}}\left[G\left(x^{\mu, n}, y^{\mu, n}\right)\right. \\
& \left.-G\left(x, y^{\mu, n}\right)\right] v_{\mu, n}^{\beta}(y) d y+\int_{\Sigma_{\mu, n}^{c} \backslash \tilde{\Sigma}_{\mu, n}}\left[G(x, y)-G\left(x^{\mu, n}, y\right)\right] v^{\beta}(y) d y \\
& \leq \int_{\Sigma_{\mu, n}}\left[G\left(x^{\mu, n}, y^{\mu, n}\right)-G\left(x, y^{\mu, n}\right)\right]\left[v^{\beta}-v_{\mu, n}^{\beta}\right](y) d y .
\end{aligned}
$$

In addition, we need the Weighted Hardy-Littlewood-Sobolev inequality:

Lemma 2.5. ([17]) Let $1<l, m<\infty, 0<\nu<n, \tau+\kappa \geq 0, \frac{1}{l}+\frac{1}{m}+\frac{\nu+\kappa+\tau}{n}=2$, and $1-\frac{1}{m}-\frac{\nu}{n}<\frac{\tau}{n}<1-\frac{1}{m}$. Then

$$
\left|\int_{\mathbb{R}^{n}} \int_{\mathbb{R}^{n}} \frac{f(x) g(y)}{|x|^{\tau}|x-y|^{\nu}|y|^{\kappa}} d x d y\right| \leq C\|f\|_{L^{m}}\|g\|_{L^{l}}
$$

with $C=C(\tau, \kappa, l, \nu, n)>0$, or equivalently,

$$
\|T g(x)\|_{L^{\gamma}}=\sup _{\|f\|_{L^{m}=1}}\langle T g(x), f(x)\rangle \leq C\|g\|_{L^{l}}
$$

with $\operatorname{Tg}(x)=\int_{\mathbb{R}^{n}} \frac{g(y)}{|x|^{\tau}|x-y|^{\nu}|y|^{\kappa}} d y, \frac{1}{l}+\frac{\nu+\kappa+\tau}{n}=1+\frac{1}{\gamma}, \frac{1}{m}+\frac{1}{\gamma}=1$.

\section{Proof of Theorem of the main results}

The techniques for the proof of Theorem 1.1 are motivated by those in [15] for a scalar problem with the Dirichlet boundary condition. We begin with two lemmas.

Lemma 3.1. Let $(\bar{u}, \bar{v}) \in L^{p}\left(\mathbb{R}_{+}^{n} \backslash B_{\epsilon}\left(z^{0}\right)\right) \times L^{q}\left(\mathbb{R}_{+}^{n} \backslash B_{\epsilon}\left(z^{0}\right)\right)$ be a positive solution of (2.3) with $\epsilon>0, p=\frac{\alpha-1}{\frac{n-2 m}{n} \alpha-1}$, and $q=\frac{\beta-1}{\frac{n-2 m}{n} \beta-1}$. If $\frac{n}{n-2 m}<\alpha, \beta \leq \frac{n+2 m}{n-2 m}$ with $\frac{\alpha+\beta}{2}<\frac{n+2 m}{n-2 m}$, or $(\bar{u}, \bar{v})$ is singular at $z^{0}$ with $\frac{\alpha+\beta}{2}=\frac{n+2 m}{n-2 m}$, then $(\bar{u}, \bar{v})$ is rotationally symmetric about any line parallel to the $x_{n}$-axis and passing through $z^{0}$.

Proof. We apply the moving-plane method in two steps:

1. Prepare to move the plane from near $x_{1}=-\infty$

Compare the values of $\left(\bar{u}_{\lambda, 1}(x), \bar{v}_{\lambda, 1}(x)\right)$ and $(\bar{u}(x), \bar{v}(x))$. Denote $w_{\lambda, 1}(x)$ $=\bar{u}(x)-\bar{u}_{\lambda, 1}(x), g_{\lambda, 1}(x)=\bar{v}(x)-\bar{v}_{\lambda, 1}(x)$. For $\lambda$ sufficiently negative, we are going to prove that

$$
w_{\lambda, 1}(x), g_{\lambda, 1}(x) \leq 0 \quad \text { for a.e. } x \in \tilde{H}_{\lambda, 1}=H_{\lambda, 1} \backslash B_{\varepsilon}\left(\left(z^{0}\right)^{\lambda}\right) .
$$

It suffices to show that both 


$$
H_{\lambda, 1}^{u}=\left\{x \in \tilde{H}_{\lambda, 1} \mid w_{\lambda, 1}(x)>0\right\} \quad \text { and } \quad H_{\lambda, 1}^{v}=\left\{x \in \tilde{H}_{\lambda, 1} \mid g_{\lambda, 1}(x)>0\right\}
$$

has measure zero.

Noticing $\left|y-z^{0}\right|>\left|y^{\lambda, 1}-z^{0}\right|$, and $(n+2 m)-\beta(n-2 m),(n+2 m)-\alpha(n-$ $2 m)>0$, by Lemma 2.2 with the mean value theorem, we have for sufficiently negative values of $\lambda$ and $x \in H_{\lambda, 1}^{u}$ that

$$
\begin{aligned}
0< & w_{\lambda, 1}(x) \\
= & \int_{H_{\lambda, 1}^{v}}+\int_{H_{\lambda, 1} \backslash H_{\lambda, 1}^{v}}\left[G\left(x^{\lambda, 1}, y^{\lambda, 1}\right)-G\left(x, y^{\lambda, 1}\right)\right]\left[\frac{\bar{v}^{\beta}(y)}{\left|y-z^{0}\right|^{(n+2 m)-\beta(n-2 m)}}\right] d y \\
& \left.-\frac{\bar{v}_{\lambda, 1}^{\beta}(y)}{\left|y^{\lambda, 1}-z^{0}\right|^{(n+2 m)-\beta(n-2 m)}}\right] d y \\
\leq & \int_{H_{\lambda, 1}^{v}}\left[G\left(x^{\lambda, 1}, y^{\lambda, 1}\right)-G\left(x, y^{\lambda, 1}\right)\right]\left[\frac{\bar{v}^{\beta}(y)}{\left|y-z^{0}\right|^{(n+2 m)-\beta(n-2 m)}}\right. \\
& -\frac{\bar{v}_{\lambda, 1}^{\beta}(y)}{\left.\left|y^{\lambda, 1}-z^{0}\right|^{(n+2 m)-\beta(n-2 m)}\right] d y} d y . \\
\leq & \beta \int_{H_{\lambda, 1}^{v}} \frac{\left[\bar{v}^{\beta-1}\left(\bar{v}-\bar{v}_{\lambda, 1}\right)\right](y)}{|x-y|^{n-2 m}\left|y-z^{0}\right|^{(n+2 m)-\beta(n-2 m)}} d y .
\end{aligned}
$$

Furthermore, by Lemma 2.5 with Hölder's inequality and $p^{*}=\frac{p}{p-1}$,

$$
\begin{aligned}
\left\|w_{\lambda}\right\|_{p, H_{\lambda, 1}^{u}} & \leq \beta \sup _{\|f\|_{L^{p^{*}}=1}} \int_{H_{\lambda, 1}^{v}} \frac{\left[\bar{v}^{\beta-1}\left(\bar{v}-\bar{v}_{\lambda, 1}\right) f\right](y)}{|x-y|^{n-2 m}\left|y-z^{0}\right|^{(n+2 m)-\beta(n-2 m)}} d y \\
& \leq C\left\|\bar{v}^{\beta-1} g_{\lambda, 1} \chi_{H_{\lambda, 1}^{v}}\right\|_{Q, \mathbb{R}^{n}} \\
& =C\left\|\bar{v}^{\beta-1} g_{\lambda, 1}\right\|_{Q, H_{\lambda, 1}^{v}} \\
& \leq C\|\bar{v}\|_{q, H_{\lambda, 1}^{v}}^{\beta-1}\left\|g_{\lambda, 1}\right\|_{p, H_{\lambda, 1}^{v}}
\end{aligned}
$$

where $\frac{1}{Q}=1+\frac{1}{p}-\frac{n-2 m+[(n+2 m)-(n-2 m) \beta]}{n}$ and throughout the paper, $C$ is used to represent positive constants independent of $x$, which may change from line to line. Similarly, we have

$$
\left\|g_{\lambda, 1}\right\|_{p, H_{\lambda, 1}^{v}} \leq C\|\bar{u}\|_{p, H_{\lambda, 1}^{u}}^{\alpha-1}\left\|w_{\lambda, 1}\right\|_{p, H_{\lambda, 1}^{u}}
$$

It follows from (3.2) and (3.3) that

$$
\left\|w_{\lambda, 1}\right\|_{p, H_{\lambda, 1}^{u}} \leq C\|\bar{u}\|_{p, H_{\lambda, 1}^{u}}^{\alpha-1}\|\bar{v}\|_{q, H_{\lambda, 1}^{v}}^{\beta-1}\left\|w_{\lambda, 1}\right\|_{p, H_{\lambda, 1}^{u}} .
$$

Since $(\bar{u}, \bar{v}) \in L^{p}\left(\mathbb{R}_{+}^{n}\right) \times L^{q}\left(\mathbb{R}_{+}^{n}\right)$, we can choose $N$ sufficiently large such that

$$
C\|\bar{u}\|_{p, H_{\lambda, 1}^{u}}^{\alpha-1}\|\bar{v}\|_{q, H_{\lambda, 1}^{v}}^{\beta-1}<\frac{1}{2}
$$

whenever $\lambda<-N$, and thus $\left\|w_{\lambda, 1}\right\|_{p, H_{\lambda, 1}^{u}}=0$ by (3.4). In the same way, $\left\|g_{\lambda, 1}\right\|_{q, H_{\lambda, 1}^{v}}=0$. This proves (3.1).

2. Move the plane to the limiting position to derive the symmetry 
Inequality (3.1) provides a starting point to move the plane $T_{\lambda, 1}$. We start from the neighborhood of $-\infty$, and move the plane to the right as long as (3.1) holds. Define

$$
\lambda_{0}=\sup \left\{\lambda \leq z_{1}^{0} \mid w_{\rho, 1}, g_{\rho, 1} \leq 0, \rho \leq \lambda \text { for a.e. } x \in \tilde{H}_{\rho, 1}\right\} .
$$

We at first prove that $\lambda_{0}=z_{1}^{0}$. Assume for contradiction that $\lambda_{0}<z_{1}^{0}$. We claim

$$
w_{\lambda_{0}, 1}(x)=g_{\lambda_{0}, 1}(x)=0 \text { a.e. in } \tilde{H}_{\lambda_{0}, 1} \text {. }
$$

Otherwise, for such a $\lambda_{0}$, e.g., the set $E_{0}=\left\{x \mid g_{\lambda_{0}, 1}(x)<0, x \in \tilde{H}_{\lambda_{0}, 1}\right\}$ possesses a positive measure. By (2.3),

$$
\begin{aligned}
& \bar{u}(x)-\bar{u}_{\lambda, 1}(x) \\
& \leq \int_{H_{\lambda, 1}}\left(G\left(x^{\lambda, 1}, y^{\lambda, 1}\right)-G\left(x, y^{\lambda, 1}\right)\right) \frac{1}{\left|y-z^{0}\right|^{(n+2 m)-\beta(n-2 m)}}\left(\bar{v}^{\beta}-\bar{v}_{\lambda, 1}^{\beta}\right)(y) d y \\
& =\int_{E_{0}}\left(G\left(x^{\lambda, 1}, y^{\lambda, 1}\right)-G\left(x, y^{\lambda, 1}\right)\right) \frac{1}{\left|y-z^{0}\right|^{(n+2 m)-\beta(n-2 m)}}\left(\bar{v}^{\beta}-\bar{v}_{\lambda, 1}^{\beta}\right)(y) d y,
\end{aligned}
$$

Consequently,

$$
w_{\lambda_{0}, 1}(x)<0 \text { a.e. in } \tilde{H}_{\lambda_{0}, 1} \text {. }
$$

Denote $\lambda_{\epsilon}=\lambda+\epsilon$ with $\epsilon>0$ to be determined. For any small $\eta>0$, choose $R$ sufficiently large such that

$$
\int_{\mathbb{R}_{+}^{n} \backslash B_{\varepsilon}\left(\left(z^{0}\right)^{\lambda_{\epsilon}}\right) \backslash B_{R}(0)}|\bar{u}|^{p}(y) d y \leq \eta .
$$

It follows from Lusin's theorem and (3.7), for any $\theta>0$, there exists a closed set $F_{\delta}$ such that $\left.w_{\lambda_{0}, 1}\right|_{F_{\delta}}$ is continuous, with $F_{\delta} \subset E:=H_{\lambda_{0}, 1} \cap B_{R}(0)$ and $m\left(E \backslash F_{\delta}\right)<\theta$. Since $w_{\lambda_{0}, 1}(x)<0$ in $\tilde{H}_{\lambda_{0}, 1}$, we know $w_{\lambda_{0}, 1}(x)<0$ in $F_{\delta}$. Choosing $\epsilon>0$ sufficiently small, we have

$$
w_{\lambda_{\epsilon}, 1}(x)<0 \quad \text { for any } x \in F_{\delta}
$$

by continuity. Denote $D_{\lambda_{\epsilon}}=\left(\tilde{H}_{\lambda_{\epsilon}, 1} \backslash H_{\lambda_{0}, 1}\right) \cap B_{R}(0)$. Then

$$
H_{\lambda_{\epsilon}, 1}^{u} \subset M:=\left(\mathbb{R}_{+}^{n} \backslash B_{\varepsilon}\left(\left(z^{0}\right)^{\lambda_{\epsilon}}\right) \backslash B_{R}(0)\right) \cup\left(E \backslash F_{\delta}\right) \cup D_{\lambda_{\epsilon}} .
$$

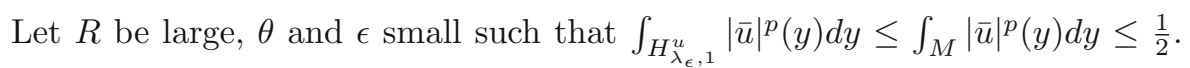
Similarly,

$$
\int_{H_{\lambda_{\epsilon}, 1}^{v}}|\bar{v}|^{q}(y) d y \leq \frac{1}{2} .
$$

By (3.4) with $\lambda=\lambda_{\epsilon}$, we can get

$$
\left\|w_{\lambda_{\epsilon}, 1}\right\|_{p, H_{\lambda_{\epsilon}, 1}^{u}} \leq C\|\bar{u}\|_{p, H_{\lambda_{\epsilon}, 1}^{u}}^{\alpha-1}\|\bar{v}\|_{q, H_{\lambda_{\epsilon}, 1}^{v}}^{\beta-1}\left\|w_{\lambda_{\epsilon}, 1}\right\|_{p, H_{\lambda_{\epsilon}, 1}^{u}} \leq \frac{1}{4}\left\|w_{\lambda_{\epsilon}, 1}\right\|_{p, H_{\lambda_{\epsilon}, 1}^{u}}
$$


which implies $\left\|w_{\lambda_{\epsilon}, 1}\right\|_{p, H_{\lambda_{\epsilon}, 1}^{u}} \equiv 0$. Thus

$$
w_{\lambda_{\epsilon}, 1}(x) \leq 0 \text { a.e. in } \tilde{H}_{\lambda_{\epsilon}, 1},
$$

and similarly,

$$
g_{\lambda_{\epsilon}, 1}(x) \leq 0 \text { a.e. in } \tilde{H}_{\lambda_{\epsilon}, 1} .
$$

This contradicts (3.5). So, the claim (3.6) is true.

For the subcritical case $\frac{n}{n-2 m}<\alpha, \beta \leq \frac{n+2 m}{n-2 m}$ with $\frac{\alpha+\beta}{2}<\frac{n+2 m}{n-2 m}$, without loss of generality, assume $\frac{n}{n-2 m}<\beta<\frac{n+2 m}{n-2 m}$. By Lemma 2.2 and $G\left(x^{\lambda_{0}, 1}, y^{\lambda_{0}, 1}\right)>G\left(x, y^{\lambda_{0}, 1}\right),\left|y-z^{0}\right|>\left|y^{\lambda_{0}, 1}-z^{0}\right|$ in $H_{\lambda_{0}, 1}$, we have

$$
\begin{aligned}
0 \equiv & \bar{u}(x)-\bar{u}_{\lambda_{0}, 1}(x) \\
& =\int_{H_{\lambda_{0}, 1}}\left[G\left(x^{\lambda_{0}, 1}, y^{\lambda_{0}, 1}\right)-G\left(x, y^{\lambda_{0}, 1}\right)\right]\left[\frac{1}{\left|y-z^{0}\right|^{(n+2 m)-\beta(n-2 m)}} \bar{v}^{\beta}\right. \\
& \left.\quad-\frac{1}{\left|y^{\lambda_{0}, 1}-z^{0}\right|^{(n+2 m)-\beta(n-2 m)}} \bar{v}_{\lambda_{0}, 1}^{\beta}\right](y) d y \\
& <\int_{H_{\lambda_{0}, 1}}\left[G\left(x^{\lambda_{0}, 1}, y^{\lambda_{0}, 1}\right)-G\left(x, y^{\lambda_{0}, 1}\right)\right] \frac{1}{\left|y-z^{0}\right|^{(n+2 m)-\beta(n-2 m)}}\left[\bar{v}^{\beta}-\bar{v}_{\lambda_{0}, 1}^{\beta}\right](y) d y \\
& \equiv 0 .
\end{aligned}
$$

This contradiction implies that $\lambda_{0}<z_{1}^{0}$ is impossible for this case.

For the critical case $\frac{\alpha+\beta}{2}=\frac{n+2 m}{n-2 m}$, with $(\bar{u}, \bar{v})$ singular at $z^{0}$, the claim (3.6) contradicts the singularity of $(\bar{u}, \bar{v})$. So, $\lambda_{0}=z_{1}^{0}$ also has to be true.

In summary, for both cases,

$$
w_{z_{1}^{0}, 1}(x), g_{z_{1}^{0}, 1}(x) \leq 0 \quad \text { for a.e } x \in H_{z_{1}^{0}, 1} \backslash B_{\varepsilon}\left(z^{0}\right),
$$

Similarly, we can move the plane from near $x_{1}=+\infty$ to the left and derive that

$$
w_{z_{1}^{0}, 1}(x), g_{z_{1}^{0}, 1}(x) \geq 0 \quad \text { for a.e } x \in H_{z_{1}^{0}, 1} \backslash B_{\varepsilon}\left(z^{0}\right) .
$$

This concludes that (3.6) holds with $\lambda_{0}=z_{1}^{0}$.

By the arbitrary of the $x_{1}$-direction chosen, we have actually shown that the solution $(u, v)$ is rotationally symmetric about any axis parallel to $x_{n}$-axis and passing through $z^{0}$.

Lemma 3.2. Let $(u, v) \in L_{\mathrm{loc}}^{p}\left(\mathbb{R}_{+}^{n}\right) \times L_{\mathrm{loc}}^{q}\left(\mathbb{R}_{+}^{n}\right)$ be a positive solution of (1.3), $p=\frac{\alpha-1}{\frac{n-2 m}{n} \alpha-1}$, and $q=\frac{\beta-1}{\frac{n-2 m}{n} \beta-1}$. Assume $\frac{n}{n-2 m}<\alpha, \beta \leq \frac{n+2 m}{n-2 m}$, if one of $\bar{u}$ and $\bar{v}$ is not singular at $z^{0}$, then $(u, v) \equiv(0,0)$.

Proof. Without loss of generality, assume that there is $z^{0} \in \partial \mathbb{R}_{+}^{n}$ such that $\bar{u}$ is not singular at $z^{0}$ ( $\bar{v}$ may be singular at $z^{0}$ or not). We have

$$
u(x)=\frac{1}{\left|x-z^{0}\right|^{n-2 m}} \bar{u}\left(\frac{x-z^{0}}{\left|x-z^{0}\right|^{2}}+z^{0}\right) .
$$

Consequently, $u(x)=O\left(|x|^{-(n-2 m)}\right),|x| \rightarrow \infty$. Together with $u \in L_{\text {loc }}^{p}\left(\mathbb{R}_{+}^{n}\right)$, we know $u \in L^{p}\left(\mathbb{R}_{+}^{n}\right)$, and so $\bar{u} \in L^{p}\left(\mathbb{R}_{+}^{n}\right)$. Moreover, $\bar{v} \in L^{q}\left(\mathbb{R}_{+}^{n} \backslash B_{\epsilon}\left(z^{0}\right)\right)$. 
The rest of the proof consists of two steps.

1 . We start from the position near $x_{n}=0$. We will show that if $\mu$ sufficiently small, then

$$
\omega_{\mu, n}(x)=\bar{u}(x)-\bar{u}_{\mu, n}(x) \leq 0 \text { a.e. in } \Sigma_{\mu, n} .
$$

Denote

$$
B_{\mu, n}^{u}=\left\{x \in \Sigma_{\mu, n} \mid \omega_{\mu, n}(x)>0\right\} .
$$

We claim that $B_{\mu, n}^{u}$ must be measure zero, provided $\mu$ sufficiently small. In fact, for any $x \in B_{\mu, n}^{u}$, similar to (3.4), we know by Lemma 2.4 that

$$
\left\|\omega_{\mu, n}\right\|_{p, B_{\mu, n}^{u}} \leq C\|\bar{u}\|_{p, B_{\mu, n}^{u}}^{\alpha-1}\|\bar{v}\|_{q, B_{\mu, n}^{v}}^{\beta-1}\left\|\omega_{\mu, n}\right\|_{p, B_{\mu, n}^{u}} .
$$

Since $(\bar{u}, \bar{v}) \in L^{p}\left(\mathbb{R}_{+}^{n}\right) \times L^{q}\left(\mathbb{R}_{+}^{n} \backslash B_{\epsilon}\left(z^{0}\right)\right)$, we can choose $\mu>0$ small enough such that

$$
C\|\bar{u}\|_{p, B_{\mu, n}^{u}}^{\alpha-1}\|\bar{v}\|_{q, B_{\mu, n}^{v}}^{\beta-1} \leq \frac{1}{2}
$$

and hence $\left\|\omega_{\mu, n}\right\|_{p, B_{\mu, n}^{u}}=0$ by (3.9).

2. The inequality (3.8) provides a starting point to move the plane $T_{\mu, n}=$ $\left\{x \in \mathbb{R}_{+}^{n} \mid x_{n}=\mu\right\}$. Now we start from the neighborhood of $x_{n}=0$, and move the plane up as long as (3.8) holds. Define $\mu_{0}=\sup \left\{\mu \mid \omega_{\rho, n} \leq 0, \rho \leq\right.$ $\mu$ a.e. in $\left.\Sigma_{\rho, n}\right\}$. If $\mu_{0}<+\infty$, similarly to (3.6), with Lemma 2.4, we can obtain

$$
\omega_{\mu_{0}, n}(x) \equiv 0 \text { a.e. in } \Sigma_{\mu_{0}, n} .
$$

This yields the contradiction that $\bar{u}(x) \equiv 0$ on the plane $\left\{x_{n}=2 \mu_{0}\right\}$. So, $\mu_{0}=+\infty$, and hence $\bar{u}$ is strictly monotonically increasing with respect to $x_{n}$. This implies that

$$
\int_{\mathbb{R}^{n-1}} \int_{a}^{\infty}\left|\bar{u}\left(x^{\prime}, a\right)\right|^{p} d x_{n} d x^{\prime}=\infty
$$

for any $a>0$, which contradicts $\bar{u} \in L^{p}\left(\mathbb{R}_{+}^{n}\right)$. We conclude $\bar{u} \equiv 0$, and so $u \equiv 0$. Moreover, $v \equiv 0$ is obtained by $(1.3)$.

Proof of Theorem 1.1. Suppose $\frac{n}{n-2 m}<\alpha, \beta \leq \frac{n+2 m}{n-2 m}$. By Lemma 3.2, if $\bar{u}$ (or $\bar{v}$ ) is not singular at a point $z^{0} \in \partial \mathbb{R}_{+}^{n}$, then $u, v \equiv 0$.

Now consider $\frac{\alpha+\beta}{2}<\frac{n+2 m}{n-2 m}$, or $\bar{u}$ and $\bar{v}$ are singular at every $z_{0} \in \partial \mathbb{R}_{+}^{n}$ with $\frac{\alpha+\beta}{2}=\frac{n+2 m}{n-2 m}$. Let $\Omega$ be a domain with a positive distance away from $z_{0}$. Then

$$
\int_{\Omega} \bar{u}^{p} d y, \int_{\Omega} \bar{v}^{q} d y<\infty .
$$

By Lemma 3.1, $(\bar{u}, \bar{v})$ is rotationally symmetric about any line parallel to $x_{n^{-}}$ axis and passing through $z^{0}$. With $X^{i}=\left(x^{i}, x_{n}\right) \in \mathbb{R}^{n-1} \times[0, \infty), i=1,2$, let $z^{0}$ be the projection of $\bar{X}=\frac{X^{1}+X^{2}}{2}$ on $\partial \mathbb{R}_{+}^{n}$. Set $Y^{i}=\frac{X^{i}-z_{0}}{\left|X^{i}-z_{0}\right|^{2}}+z^{0}$, $i=1,2$. From the above arguments, we have $\bar{u}\left(Y^{1}\right)=\bar{u}\left(Y^{2}\right), \bar{v}\left(Y^{1}\right)=\bar{v}\left(Y^{2}\right)$, since $\left|Y^{1}-z^{0}\right|=\left|Y^{2}-z^{0}\right|$. Hence $u\left(X^{1}\right)=u\left(X^{2}\right), v\left(X^{1}\right)=v\left(X^{2}\right)$. This 
implies that $(u, v)$ only depends on the $x_{n}$-variable. Denote $u(x)=u\left(x_{n}\right)$, $v(x)=v\left(x_{n}\right)$ for simplicity. For fixed $x \in \mathbb{R}_{+}^{n}$, choose $R$ large enough such that $\left|x_{n}\right|<\frac{R}{2}$. We have

$$
u\left(x_{n}\right) \geq \int_{\mathbb{R}_{+}^{n} \backslash B_{R}(0)}\left(\frac{1}{|x-y|^{n-2 m}}-\frac{1}{|\bar{x}-y|^{n-2 m}}\right) v^{\beta}\left(y_{n}\right) d y .
$$

By the mean value formula, we know for $y \in \mathbb{R}_{+}^{n} \backslash B_{R}(0)$ that

$$
\begin{aligned}
\frac{1}{|x-y|^{n-2 m}}-\frac{1}{|\bar{x}-y|^{n-2 m}} & =(2 m-n) \frac{\left(\xi_{1}-y_{1}, \ldots, \xi_{n}-y_{n}\right) \cdot\left(0, \cdots, 2 x_{n}\right)}{|\xi-y|^{n-2 m+2}} \\
& \geq \frac{c}{|y|^{n-2 m+2}},
\end{aligned}
$$

with $c=c\left(x_{n}, R\right)>0$. Set $\left|y^{\prime}\right|=r,\left|y_{n}\right|=a$. We have

$$
\begin{aligned}
u\left(x_{n}\right) & \geq \int_{\mathbb{R}_{+}^{n} \backslash B_{R}(0)} c \frac{1}{|y|^{n-2 m+2}} v^{\beta}(y) d y \\
& =c \int_{R}^{\infty} \frac{v^{\beta}\left(y_{n}\right)\left|y_{n}\right|^{2 m-2}}{\left|y_{n}\right|} d y_{n} \int_{R}^{\infty} \frac{\left(\frac{r}{a}\right)^{n-2}}{\left[\left|\frac{r}{a}\right|^{2}+1\right]^{\frac{n}{2}}} d\left(\frac{r}{a}\right) \\
& \geq c \int_{R}^{\infty} \frac{v^{\beta}\left(y_{n}\right)}{y_{n}^{2-2 m}} d y_{n} .
\end{aligned}
$$

Due to the integer $m \in(0, n / 2)$, there exists a sequence $y_{n}^{i} \rightarrow \infty$ as $i \rightarrow \infty$, such that $\lim _{i \rightarrow \infty} v\left(y_{n}^{i}\right)=0$.

For simplicity, denote $u\left(x_{n}\right)=u(t), v\left(x_{n}\right)=v(t)$.

Notice $m \in \mathbb{N}$ satisfying $0<2 m<n$. If $m=2 k$ with $k \in \mathbb{N}$, we have

$$
\begin{aligned}
u^{(2 m)}(t)=(-\Delta)^{m} u(x) & =\int_{\mathbb{R}_{+}^{n}}(-\Delta)^{m}\left(\frac{1}{|x-y|^{n-2 m}}-\frac{1}{|\bar{x}-y|^{n-2 m}}\right) v^{\beta}(y) d y \\
& =v^{\beta}(x)>0,
\end{aligned}
$$

which implies $u^{(2 m-1)}(t)$ is increasing.

We claim that $u^{(2 m-1)}(t) \leq 0$. Otherwise, there is $t_{0}>0$ such that $u^{(2 m-1)}\left(t_{0}\right)>0$, and thus

$$
u^{(2 m-1)}(t) \geq u^{(2 m-1)}\left(t_{0}\right)>0 \text { for } t \geq t_{0}>0 .
$$

Integrating several times, and then letting $t \rightarrow \infty$, we have $u(t) \rightarrow \infty$. This contradicts the fact that $u\left(y_{n}^{i}\right) \rightarrow 0$. The claim implies $u^{(2 m-2)}(t)$ is nonincreasing.

By deduction, we derive that $u(t)$ is nonincreasing. Together with the positivity of $u$ and $u(0)=0$, we conclude $u \equiv 0$. Therefore, $v \equiv 0$ as well due to the second equation of (1.3).

If $m=2 k-1$ with $k \in \mathbb{N}$, we can show by a similar procedure that $v(t)$ is nondecreasing. By (3.10), we have

$+\infty>u(x)=u\left(x_{n}\right) \geq c \int_{R}^{\infty} \frac{v^{\beta}\left(y_{n}\right)}{y_{n}^{2-2 m}} d y_{n} d y_{n} \geq c v^{\beta}(R) \int_{R}^{\infty} \frac{1}{y_{n}^{2-2 m}} d y_{n} d y_{n}=+\infty$, and hence $v \equiv 0$. In addition, $u \equiv 0$ by (1.3). 
Finally, it suffices to show the equivalence between the differential system (1.1) and the integral system (1.3) (Theorem 1.2). The required proof is identical mutatis mutandis to the same result for the single equation case.

It is easy to see that the higher-order PDEs problem (1.1) can be rewritten as the following second-order system

$\left\{\begin{array}{l}-\Delta v_{i}=v_{i+1},\left.v_{i}\right|_{\partial \mathbb{R}_{+}^{n}}=0, i=0,1, \ldots, m-1, \text { with } v_{0}=u, v_{m}=v^{\beta}, \\ -\Delta u_{i}=u_{i+1},\left.u_{i}\right|_{\partial \mathbb{R}_{+}^{n}}=0, i=0,1, \ldots, m-1, \text { with } u_{0}=v, u_{m}=u^{\alpha} .\end{array}\right.$

On the other hand, rewrite the integral system (1.3) as

$$
\left\{\begin{array}{l}
v_{i}=\int_{\mathbb{R}_{+}^{n}} G(x, y, 2) v_{i+1}(y) d y, i=0,1, \ldots, m-1, \text { with } v_{0}=u, v_{m}=v^{\beta}, \\
u_{i}=\int_{\mathbb{R}_{+}^{n}} G(x, y, 2) u_{i+1}(y) d y, i=0,1, \ldots, m-1, \text { with } u_{0}=v, u_{m}=u^{\alpha},
\end{array}\right.
$$

where $G(x, y, 2)$ is defined by $(2.1)$ with $m=1$.

Consequently, by using the technique introduced by Chen and Fang [9] for the scalar case of higher-order equations, we can establish the equivalence between the $2 m$ second-order elliptic equations (3.11) and the $2 m$ integral system (3.12). We omit the details.

\section{References}

[1] Berestycki, H., Capuzzo Dolcetta, I., Nirenberg, L.: Problèmes elliptiques indéfinis et théorèmes de Liouville non linèaires. C. R. Acad. Sci. Paris Série. I Math. 317, 945-950 (1993)

[2] Berestycki, H., Capuzzo Dolcetta, I., Nirenberg, L.: Superlinear indefinite elliptic problems and nonlinear Liouville theorems. Topol. Methods Nonlinear Anal. 4, 59-78 (1994)

[3] Birindelli, I., Mitidieri, E.: Liouville theorems for elliptic inequalities and applications. Proc. Roy. Soc. Edinburgh 128, 1217-1247 (1998)

[4] Brason, T.: Differential operators canonically associated to a conformal structure. Math. Scand. 2, 293-345 (1985)

[5] Cao, L., Chen, W.: Liouville type theorems for poly-harmonic Navier problems. Discrete Contin. Dyn. Syst. 33, 3937-3955 (2013)

[6] Cao, L., Dai, Z.: A Liouville-type theorem for an integral equation on a halfspace $\mathbb{R}_{+}^{n}$. J. Math. Anal. Appl. 389, 1365-1373 (2012)

[7] Caristi, G., D'Ambrosio, L., Mitidieri, E.: Representation formula for solutions to some classes of higher order systems and related Liouville theorems. Milan J. Math. 76, 27-67 (2008)

[8] Chen, W., Li, C., Ou, B.: Classification of solutions for an integral equation. Comm. Pure Appl. Math. 59, 330-343 (2006) 
[9] Chen, W., Fang, Y., Li, C.: Super poly-harmonic property of solutions for Navier boundary problems on a half space. J. Funct. Anal. 265, 1522-1555 (2013)

[10] Chen, Z., Zhao, Z.: Potential theory for elliptic systems. Ann. Probab. 24, 293319 (1996)

[11] de Figueiredo, D., Felmer, P.: A Liouville-type theorem for elliptic systems. Ann. Scuola Norm. Sup. Pisa CI. Sci. 21, 387-397 (1994)

[12] Djadli, Z., Malchiodi, A., Almedou, M.: Prescribing a fourth order conformal invariant on the standard sphere. II. Blow up analysis and applications. Ann. Sc. Norm. Super. Pisa CI. Sci. 1, 387-434 (2002)

[13] Domingos, A.R., Guo, Y.: A note on a Liouville type result for a system of fourth-order equations in $\mathbb{R}^{N}$. Electron. J. Differ. Equ. 99, 1-20 (2002)

[14] Esposito, P., Robert, F.: Mountain pass critical points for Paneitz-Branson operators. Calv. Var. Partial Differ. Equ. 15, 493-517 (2002)

[15] Fang, Y., Chen, W.: A Liouville type theorem for poly-harmonic Dirichlet problems in a half space. Adv. Math. 229, 2835-2867 (2012)

[16] Gidas, B., Spruck, J.: A priori bounds for positive solutions of nonlinear elliptic equations. Comm. Partial Differ. Equ. 6, 525-598 (1981)

[17] Jin, C., Li, C.: Symmetry of solutions to some integral equations. Proc. Am. Math. Soc. 134, 1661-1670 (2005)

[18] Lenhart, S., Belbas, S.: A system of nonlinear partial differential equations arising in the optimal control of stochastic systems. SIAM J. Appl. Math. 43, 465475 (1983)

[19] Li, C., Ma, L.: Uniqueness of positive bound states to shrödinger systems with critical exponents. SIAM J. Math. Anal. 40:1049-1057 (2008)

[20] Liu, J., Guo, Y., Zhang, Y.: Liouville-type theorem for polyharmonic systems in $\mathbb{R}^{n}$. J. Differ. Equ. 225, 685-709 (2006)

[21] Lu, G., Wang, P., Zhu, J.: Liouville-type theorems and decay estimates for solutions to higher order elliptic equations. Ann. I. H. Poincaré-AN 29, 653665 (2012)

[22] Ma, L., Chen, D.: A Liouville type theorem for an integral system. Commun. Pure Appl. Anal. 5, 855-859 (2006)

[23] Mitidieri, E.: A Rellich type identity and applications. Comm. Partial Differ. Equ. 18, 125-151 (1993)

[24] Mitidieri, E.: Nonexistence of positive solutions of semilinear elliptic systems in $\mathbb{R}^{n}$. Differ. Integral Equ. 9, 465-479 (1996)

[25] Nazarov, S., Sweers, G.: A hinged plate equation and iterated Dirichlet Laplace operator on domanins with concave corners. J. Differ. Equ. 233, 151-180 (2007) 
[26] Peletier, L.: Nonlinear eigenvalue problems for higher-order model equations. Handbook of Differential Equations, Stationary Partial Differential Equations, Volume 3, Chapter 7, Ed. M. Chipot and P. Quittner, Elsevier (2006)

[27] Peter, P., Quittner, P., Souplet, Ph.: Singularity and decay estimates in superlinear problems via Liouville-type theorems. Part I: elliptic equations and systems. Duke Math. J. 139, 555-579 (2007)

[28] Peletier, L.; Troy, W.; Patterns, S.: Higher order models in physics and mechanics, Progress in Nonlinear Differential Equations and their Applications. Birkhauser Boston, Inc., Boston, MA, 45 (2001)

[29] Reichel, W., Weth, T.: A prior bounds and a Liouville theorem on a half-space for higher-order elliptic Dirichlet problems. Math. Z. 261, 805-827 (2009)

[30] Reichel, W., Weth, T.: Existence of solutions to nonlinear, subcritical higherorder elliptic dirichlet problems. J. Differ. Equ. 248, 1866-1878 (2010)

[31] Serrin, J., Zou, H.: Nonexistence of positive solutions of Lane-Emden systems. Differ. Integral Equ. 9, 635-653 (1996)

[32] Sirakov, B.: Existence results and a priori bounds for higher order elliptic equations and systems. J. Math. Pures Appl. 89, 114-133 (2008)

[33] van den Berg, J.B.: The phase-plane picture for a class of fourth-order conservative differential equations. J. Differ. Equ. 161, 110-153 (2000)

[34] Wei, J., Xu, X.: Classification of solutions of higher order conformally invariant equations. Math. Ann. 313, 207-228 (1999)

[35] Zhang, Y.: A Liouville type theorems for poly-harmonic elliptic systems. Electron. J. Differ. Equ. 99, 1-20 (2002)

Weiwei Zhao, Jinge Yang and Sining Zheng

School of Mathematical Sciences, Dalian University of Technology

Dalian 116024

People's Republic of China

e-mail: snzheng@dlut.edu.cn

Weiwei Zhao

e-mail: zhaoweiwei336699@163.com

Jinge Yang

Department of Science

Nanchang Institute of Technology

Nanchang 330099

People's Republic of China

e-mail: sundayday@163.com

Received: 11 December 2013.

Accepted: 31 July 2014. 